\title{
Photogrammetry experiments with a model eye
}

\author{
A. R. ROSENTHAL, ${ }^{1}$ D. G. FAlCONER, ${ }^{2}$ AND I. PIEPER ${ }^{1}$ \\ From the ${ }^{1}$ Division of Ophthalmology, Stanford University Medical Center, and from the \\ ${ }^{2}$ Artificial Intelligence Center, Stanford Research Institute, International, USA
}

SUMMARY Digital photogrammetry was performed on stereophotographs of the optic nerve head of a modified Zeiss model eye in which optic cups of varying depths could be simulated. Experiments were undertaken to determine the impact of both photographic and ocular variables on the photogrammetric measurements of cup depth. The photogrammetric procedure tolerates refocusing, repositioning, and realignment as well as small variations in the geometric position of the camera. Progressive underestimation of cup depth was observed with increasing myopia, while progressive overestimation was noted with increasing hyperopia. High cylindrical errors at axis $90^{\circ}$ led to significant errors in cup depth estimates, while high cylindrical errors at axis $180^{\circ}$ did not materially affect the accuracy of the analysis. Finally, cup depths were seriously underestimated when the pupil diameter was less than $5.0 \mathrm{~mm}$.

A digital photogrammetric technique has been used to quantitate optic nerve head cupping employing stereophotographs. ${ }^{1}$ Comparative studies indicated that the most reproducible results were obtained when simultaneous stereophotographs were taken with the Donaldson stereoscopic fundus camera. ${ }^{2}$ Since there is no accurate method for comparing the actual geometry with the photogrammetric estimates in the human eye, we thought it would be useful to use a model eye in which a cup with known dimensions could be photographed and analysed. In addition experiments were undertaken to determine the impact of photographic and ocular variables on the photogrammetric estimates of a known cup depth in photographs taken with the Donaldson stereo fundus camera.

\section{Materials and methods}

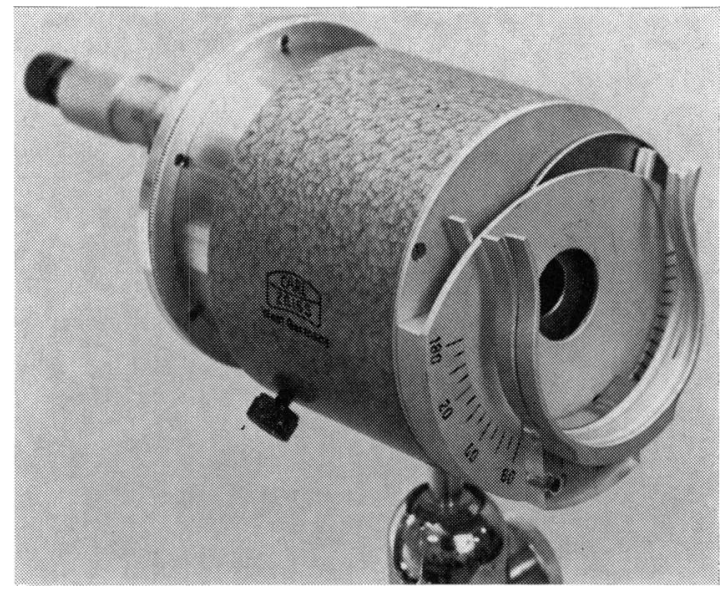

Fig. 1a Zeiss model eye (front view).

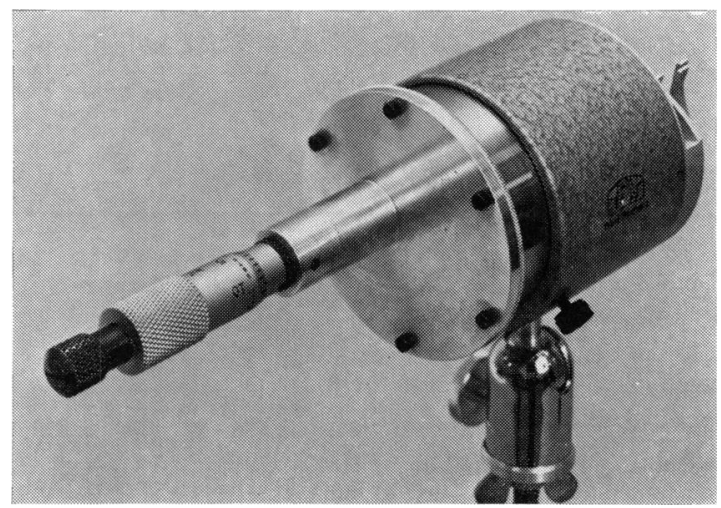

Fig. 1b Zeiss model eye (back view).
Correspondence to Dr A. R. Rosenthal, Division of Ophthalmology, Stanford University Medical Center, Stanford, CA 94305, USA. 


\section{CAMERA}

The Donaldson stereoscopic fundus camera was used to take all the disc stereophotographs. ${ }^{3}$ The approximate specifications of the Donaldson stereoscopic fundus camera used in this study are displayed in Table 2.

\section{PHOTOGRAPHIC PROCEDURE}

The modified model eye was mounted securely on the headrest ring of the Donaldson fundus camera with an aluminium bar and clamp. Stereopairs were taken in the usual way after centring the optic disc in the camera's viewfield. Uniform illumination of the nerve head was obtained by shifting the camera horizontally and vertically until the illumination light entered the model eye's pupil in a symmetric configuration. Spurious reflections were eliminated and exact focus was achieved before photographs were taken.

All photographs were taken with the superior

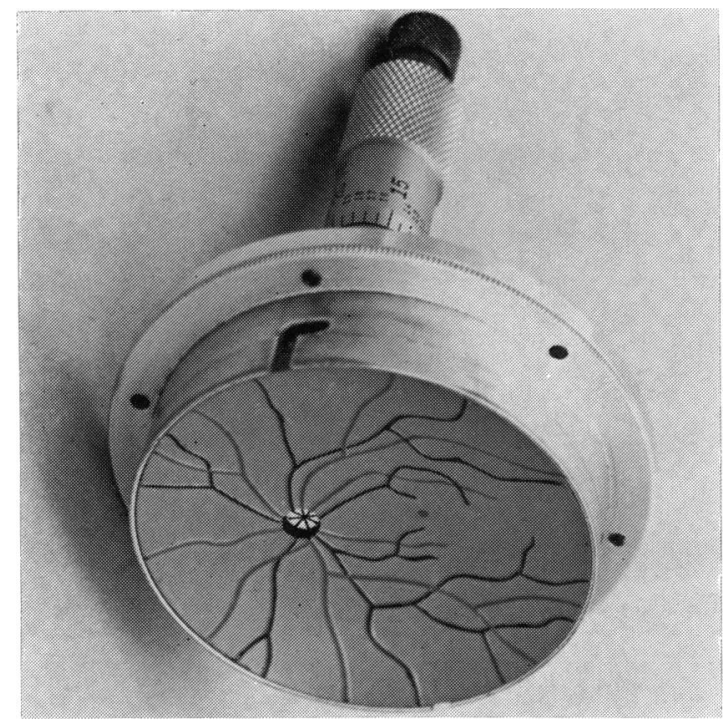

Fig. 2 Simulated optic disc and retina in Zeiss model eye.

Table 1 Zeiss model eye parameters

\begin{tabular}{|c|c|}
\hline Focal length of lens: & $50 \mathrm{~mm}$ \\
\hline Optic cup diameter: & $3.75 \mathrm{~mm}$ \\
\hline Cup depth: & $\begin{array}{l}\text { Variable from } 1 \mathrm{~mm} \text { cup elevation to } \\
9 \mathrm{~mm} \text { cup depression }\end{array}$ \\
\hline Pupil size: & Variable from 4-8 $\mathrm{mm}$ \\
\hline Refractive error: & Variab'e from -5 myopia to +6 hyperopia \\
\hline Nominal cup depth: & $\begin{array}{l}1.27 \mathrm{~mm} \text { used for all experiments, except } \\
\text { the one in which variations in depth } \\
\text { were measured }\end{array}$ \\
\hline
\end{tabular}

Table 2 Specifications and measurements of Donaldson stereofundus camera

\begin{tabular}{lc}
\hline Stereobase: & 2.50 and $2.875 \mathrm{~mm}$ \\
Focal length of camera: & $\begin{array}{c}8.67 \mathrm{~mm} \text { (at } 6 \text { times camera } \\
\text { magnification) }\end{array}$ \\
Focal length of objective lens: & $\begin{array}{c}50 \mathrm{~mm} \text { (corresponds with that of } \\
\text { model eye) }\end{array}$ \\
Focal length of relay lens: & $\begin{array}{l}95 \mathrm{~mm} \text { (approximate) } \\
\text { Image magnification on film: }\end{array}$ \\
$\begin{array}{l}1.70 \text { (at } 6 \text { times camera } \\
\text { magnification) }\end{array}$ \\
\hline
\end{tabular}

and inferior portions of the retinal surface in best focus. Accordingly, the nerve head vessels tended to go out of focus on the deepest cups. The nominal cup depth was set at $1.27 \mathrm{~mm}$ for studying most of the variables. The retina-to-film magnification was approximately $1 \cdot 7$. Stereobase plates with 4.5 and $5.0 \mathrm{~mm}$ apertures $(5.0$ and $5.75 \mathrm{~mm}$ centre-to-centre separation respectively) were used.

Kodachrome II film was used for all the photographs, and the resulting pictures were hand mounted in 2 inch $\times 2$ inch $(5 \times 5 \mathrm{~cm})$ slides.

\section{VARIABLES STUDIED}

Cup depth. The depth of the cup was varied by repositioning the micrometer so that depths varying from $0.00 \mathrm{~mm}$ to $3.81 \mathrm{~mm}$ in $0.64 \mathrm{~mm}$ increments were obtained.

Pupil size. Extra positions in the pupil wheel were drilled yielding pupil sizes of $4-8 \mathrm{~mm}$ in $1 \mathrm{~mm}$ increments.

Refractive error. Hyperopia and myopia of 5 dioptres in $1 / 2$ dioptre increments were simulated by moving the retinal surface fore and aft respectively relative to the model eye's lens. Stereopairs were taken at $1 / 2$ dioptre intervals to ensure a well defined curve of measurement error versus refractive error. Astigmatism was simulated by placing both plus and minus cylindrical lenses of 1 and 2 dioptres at both $90^{\circ}$ and $180^{\circ}$ in the model eye's lens holder.

Refocusing/realigning/repositioning effects. Stereopairs were taken of the model eye after the following manoeuvres were performed either separately or in combination: refocusing, realigning, and repositioning of the fundus camera.

Elevational and azimuthal effects. Stereophotographs were taken of the model eye after rotating the fundus camera from the determined centered position $1 / 4$ and $1 / 2$ disc diameters in both the horizontal and vertical directions.

Horizontal and vertical translational effects. Stereophotographs were taken of the model eye after the fundus camera was moved 1 and $2 \mathrm{~mm}$ right and left and up and down from its nominally centred position. 
DIGITAL PHOTOGRAMMETRIC

PROCEDURE

The developed and mounted stereopairs were digitised with an electronic film scanner. The recording matrix used was $256 \times 256$ pixels with a pixel-to-pixel spacing of $10 \mu \mathrm{m}$. This matrix and spacing limited the scanning operation to $1 / 3$ of the disc area. Accordingly the superior portion of the optic disc and the adjoining portion of the retinal surface were selected for digitisation.

A new program, MODEL, was abstracted from our traditional one, STEREO. Since a full-height map was unnecessary, this algorithm made a single determination of the absolute height of the optic cup relative to the retinal surface.

The depth sensitivity was defined as the smallest change in cup depth which can be confidently detected by the procedure. This distance is directly proportional to the pixel spacing and is a function of the mask geometry and the focal lengths of the camera and the model eye. In the present study the depth sensitivity was $0.105 \mathrm{~mm}$ and $0.120 \mathrm{~mm}$ when the $5.75 \mathrm{~mm}$ and $5.00 \mathrm{~mm}$ masks were respectively employed.

\section{Results}

Measured vs. actual depth. In order to determine how accurately the photogrammetric procedure measures different cup sizes, stereopairs were taken at various cup depths with no changes in the fundus camera conditions. Three measurements were made at each cup depth. The eye was set at emmetropia, and the $5.75 \mathrm{~mm}$ mask was used throughout the photographic session. Table 3 shows how the photogrammetric procedure measures known variations in cup depth, while Fig. 3 graphically displays these results.

\section{OPTIC VARIABLES}

Pupillary diameter. To determine the impact of pupil size on cup depth measurements 3 stereopairs were taken of the nerve head of the model eye at different pupil diameters with the fundus camera left in fixed position. In this set of photographs the eye was set at emmetropia and the $5.0 \mathrm{~mm}$ mask was employed, except at the $3 \mathrm{~mm}$ pupil, when the $5.75 \mathrm{~mm}$ mask was used.

Results are tabulated in Table 4 and displayed graphically in Fig. 4. Note that the measured depth of the optic cup decreased when pupil diameters were less than $5 \mathrm{~mm}$. By means of the correction factor detailed in a previous communication ${ }^{4}$ a corrected cup depth is calculated for each pupil size and is included in Table 4. This corrected cup depth when pupil size was less than $5 \mathrm{~mm}$ is comparable to the actual depth.
Refractive error. Three stereophotographs were taken of the optic nerve head of the model eye at varying degrees of hyperopia and myopia. For all the photographs the $5.0 \mathrm{~mm}$ mask was employed. To correct for refraction effects a theoretical analysis was undertaken, and the formulas produced from this analysis ${ }^{4}$ were used to correct the measured cup depths. Table 5 displays the effect of refractive error on cup depth measurements.

Note that the measured cup depth decreases dramatically with increasing myopia and increases dramatically with increasing hyperopia. Fig. 5 shows graphically how the cup depth estimates change with alterations in refractive error when the Donaldson camera is used to photograph either the model eye or the human eye. The former curve

Table 3 Accuracy of photogrammetric procedure

\begin{tabular}{ll}
\hline $\begin{array}{l}\text { Actual cup depth } \\
(\mathrm{mm})\end{array}$ & $\begin{array}{l}\text { Measured cup depth } \\
(\mathrm{mm}) \\
\text { Mean } \pm S D, n=3\end{array}$ \\
\hline 0.00 & $0.26 \pm 0.07$ \\
0.64 & $0.63 \pm 0.02$ \\
1.27 & $1.26 \pm 0.11$ \\
1.90 & $1.98 \pm 0.07$ \\
2.54 & $2.55 \pm 0.04$ \\
3.18 & $3.13 \pm 0.11$ \\
3.81 & $3.44 \pm 0.27$ \\
\hline
\end{tabular}

Depth sensitivity $=0.105 \mathrm{~mm}$.

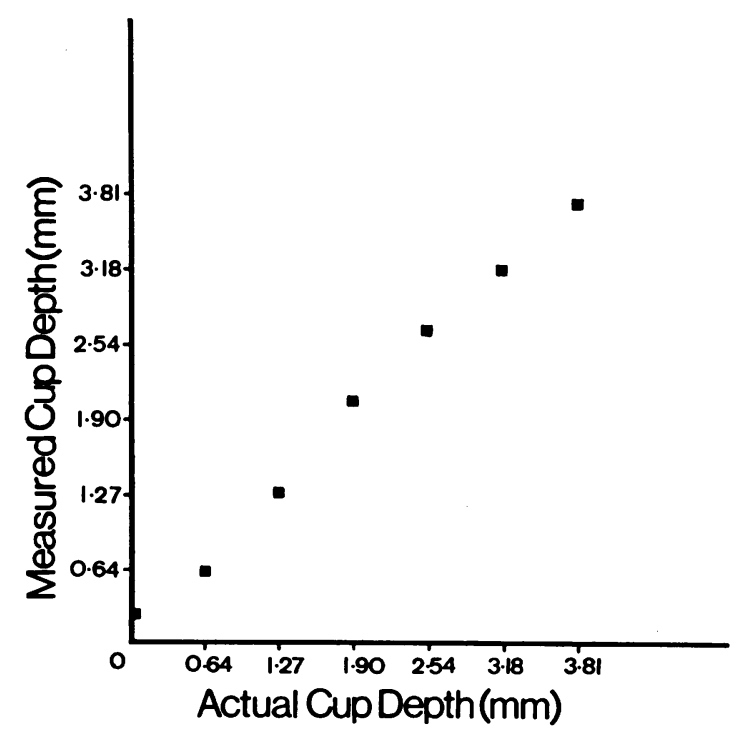

Fig. 3 Comparison of measured cup depth by the photogrammetric procedure with actual cup depth of the Zeiss model eye. 
is determined from these experiments, while the latter curve is established from theoretical considerations. $^{4}$

Astigmatism. One stereopair was taken of the optic nerve of the model eye after cylindrical lenses were mounted in the lens holder of the model eye. The $5.0 \mathrm{~mm}$ mask was used in the camera. Table 6 details the results of the photogrammetrically measured cup depths as compared with the actual depths.

Though only one stereopair was taken with each astigmatic error, it appears that high horizontal and vertical cylinders affect cup depth estimates differently. High positive vertical axis cylinders appear to underestimate cup depth, while high negative vertical axis cylinders seem to have the opposite effect of overestimating the cup depth. Lower

Table 4 Cup depth and pupil size

\begin{tabular}{llll}
\hline $\begin{array}{l}\text { Pupil } \\
\text { diameter } \\
(\mathrm{mm})\end{array}$ & $\begin{array}{l}\text { Actual } \\
\text { cup depth } \\
(\mathrm{mm})\end{array}$ & $\begin{array}{l}\text { Measured } \\
\text { cup depth } \\
(\mathrm{mm}) \\
n=3\end{array}$ & $\begin{array}{l}\text { Corrected } \\
\text { cup depth } \\
(\mathrm{mm})\end{array}$ \\
\hline 3 & 1.27 & 0.70 & 1.20 \\
4 & 1.27 & 1.05 & 1.31 \\
5 & 1.27 & 1.28 & 1.28 \\
6 & 1.27 & 1.30 & 1.30 \\
7 & 1.27 & 1.25 & 1.25 \\
8 & 1.27 & 1.27 & 1.27 \\
\hline
\end{tabular}

Depth sensitivity $=0.120 \mathrm{~mm}$ except with $3 \mathrm{~mm}$ pupil, where it equals $0 \cdot 105 \mathrm{~mm}$.

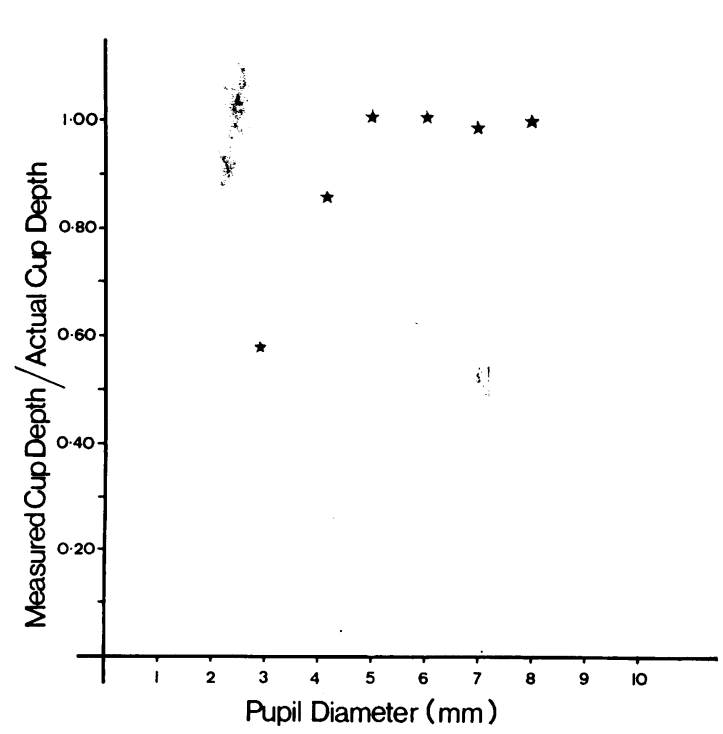

Fig. 4 Effect of pupil diameter on measured cup depth in the Zeiss model eye.
Table 5 Effect of refractive error on cup depth measurements

\begin{tabular}{|c|c|c|c|c|}
\hline & $\begin{array}{l}\text { Refractive } \\
\text { error } \\
\text { (dioptres) }\end{array}$ & $\begin{array}{l}\text { Actual } \\
\text { cup depth } \\
(\mathrm{mm})\end{array}$ & $\begin{array}{l}\text { Measured } \\
\text { cup depth } \\
(\mathrm{mm}) \\
n=3\end{array}$ & $\begin{array}{l}\text { Corrected } \\
\text { cup depth } \\
(\mathrm{mm})\end{array}$ \\
\hline \multirow{10}{*}{ Myopia } & $5 \cdot 0$ & $1 \cdot 27$ & 0.48 & 0.92 \\
\hline & $4 \cdot 5$ & $1 \cdot 27$ & 0.48 & 0.86 \\
\hline & $4 \cdot 0$ & $1 \cdot 27$ & $0 \cdot 24$ & 0.40 \\
\hline & $3 \cdot 5$ & $1 \cdot 27$ & Not done & Not done \\
\hline & $3 \cdot 0$ & $1 \cdot 27$ & 0.48 & $0 \cdot 70$ \\
\hline & $2 \cdot 5$ & $1 \cdot 27$ & 0.72 & 1.00 \\
\hline & $2 \cdot 0$ & $1 \cdot 27$ & 0.60 & 0.75 \\
\hline & $1 \cdot 5$ & $1 \cdot 27$ & 0.72 & 0.80 \\
\hline & $1 \cdot 0$ & $1 \cdot 27$ & 0.84 & 0.93 \\
\hline & 0.5 & $1 \cdot 27$ & 0.96 & 0.99 \\
\hline Emmetropia & 0 & $1 \cdot 27$ & $1 \cdot 20$ & $1 \cdot 20$ \\
\hline \multirow[t]{10}{*}{ Hypertropia } & $0 \cdot 5$ & $1 \cdot 27$ & $1 \cdot 20$ & 1.05 \\
\hline & $1 \cdot 0$ & $1 \cdot 27$ & $1 \cdot 32$ & $1 \cdot 12$ \\
\hline & $1 \cdot 5$ & $1 \cdot 27$ & $1 \cdot 20$ & 0.95 \\
\hline & $2 \cdot 0$ & $1 \cdot 27$ & $2 \cdot 04$ & 1.53 \\
\hline & $2 \cdot 5$ & $1 \cdot 27$ & $1 \cdot 80$ & $1 \cdot 28$ \\
\hline & $3 \cdot 0$ & $1 \cdot 27$ & 1.92 & $1 \cdot 28$ \\
\hline & $3 \cdot 5$ & $1 \cdot 27$ & $2 \cdot 28$ & $1 \cdot 46$ \\
\hline & $4 \cdot 0$ & $1 \cdot 27$ & $2 \cdot 76$ & 1.66 \\
\hline & $4 \cdot 5$ & $1 \cdot 27$ & $2 \cdot 28$ & $1 \cdot 32$ \\
\hline & $5 \cdot 0$ & $1 \cdot 27$ & $2 \cdot 40$ & $1 \cdot 29$ \\
\hline
\end{tabular}

Depth sensitivity $=0.120 \mathrm{~mm}$.

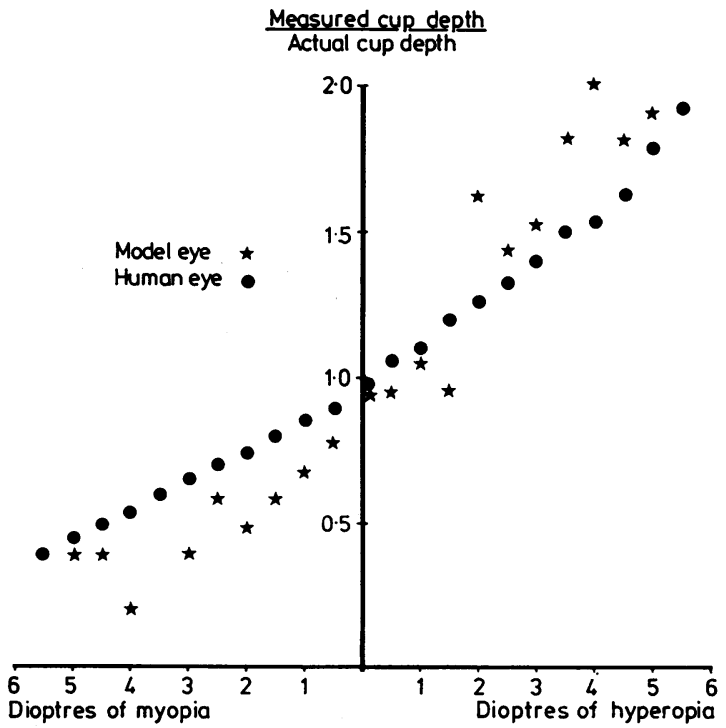

Fig. 5 Effect of refractive error on measured cup depth in the Zeiss model eye. 
Table 6 Cylindrical errors

\begin{tabular}{|c|c|c|c|}
\hline \multicolumn{2}{|l|}{ Cylinder } & \multicolumn{2}{|l|}{ Cup depth } \\
\hline Dioptres & $\begin{array}{l}\text { Axis } \\
\text { (degrees) }\end{array}$ & $\begin{array}{l}\text { Actual cup } \\
\text { depth }(\mathrm{mm})\end{array}$ & $\begin{array}{l}\text { Measured cup } \\
\text { depth }(\mathrm{mm}) \\
n=1\end{array}$ \\
\hline \multicolumn{2}{|c|}{ Emmetropia } & $1 \cdot 27$ & $1 \cdot 20$ \\
\hline$+2 \cdot 0$ & 90 & $1 \cdot 27$ & 0.84 \\
\hline$+1 \cdot 0$ & 90 & $1 \cdot 27$ & $1 \cdot 20$ \\
\hline$-1 \cdot 0$ & 90 & $1 \cdot 27$ & $1 \cdot 32$ \\
\hline$-2 \cdot 0$ & 90 & $1 \cdot 27$ & 1.44 \\
\hline$+2 \cdot 0$ & 180 & $1 \cdot 27$ & $1 \cdot 20$ \\
\hline+1.0 & 180 & $1 \cdot 27$ & $1 \cdot 32$ \\
\hline$-1 \cdot 0$ & 180 & $1 \cdot 27$ & $1 \cdot 20$ \\
\hline$-2 \cdot 0$ & 180 & $1 \cdot 27$ & $1 \cdot 32$ \\
\hline
\end{tabular}

Depth sensitivity $=0.120 \mathrm{~mm}$.

Table 7 Effect of refocusing, realignment, and repositioning on cup depth of model eye

\begin{tabular}{lll}
\hline Type of camera adjustment & $\begin{array}{l}\text { Actual cup } \\
\text { depth }(\mathrm{mm})\end{array}$ & $\begin{array}{l}\text { Measured cup } \\
\text { depth }(\mathrm{mm}) \\
\text { Mean } \pm \text { SD, } \\
n=5\end{array}$ \\
\hline No adjustments* & 1.27 & $1.27 \pm 0.086$ \\
Camera refocused* & 1.27 & $1.21 \pm 0.092$ \\
Camera realigned** & 1.27 & $1.23 \pm 0.087$ \\
Camera realigned and refocused*** & 1.27 & $1.28 \pm 0.079$ \\
$\begin{array}{l}\text { Camera repositioned, realigned, } \\
\text { and refocused** }\end{array}$ & 1.27 & $1.20 \pm 0.079$ \\
\hline $\begin{array}{l}\text { *Depth sensitivity }=0.105 \mathrm{~mm} . \\
\text { *"Depth sensitivity }=0.120 \mathrm{~mm} .\end{array}$ &
\end{tabular}

cylindrical errors do not seem to alter the accuracy of the analysis. Neither high nor low horizontal axis cylinders appear to have a systematic effect on the photogrammetric estimation of cup depth.

\section{PHOTOGRAPHIC VARIABLES}

Realignment, refocusing, repositioning. Five stereopairs were taken of the model eye optic disc after the following manoeuvres were performed with the fundus camera: refocusing, realignment, realignment and refocusing, and finally repositioning, realignment, and refocusing. The first set of photographs was taken with the $5.75 \mathrm{~mm}$ mask, while the latter 3 groups of photographs were taken with the $5.0 \mathrm{~mm}$ mask. The eye was set at emmetropia for all the photographs. Table 7 details the results of the digital photogrammetric analyses. No significant alterations in cup depth measurements were produced by these manoeuvres.

Elevational and azimuthal effects. One stereopair of the model eye disc was taken after rotating the fundus camera by $1 / 4$ disc diameter (1 step rotation) and 1/2 disc diameter ( 2 step rotation) in both horizontal and vertical directions. All photographs were taken in the emmetropic position using the $5.75 \mathrm{~mm}$ mask. Poor agreement between actual and measured cup depth occurred as a result of these manoeuvres. Table 8 displays the results of the digital photogrammetric analyses.

Horizontal and vertical translational effects on optic cup depth. One stereopair was taken of the model eye disc after the fundus camera was moved right and left and up and down by 1 and $2 \mathrm{~mm}$ from the nominally centered position. The photographs were taken in the emmetropic eye with the $5.75 \mathrm{~mm}$ mask. Table 9 displays the results of these analyses. Though only one stereopair was analysed, the estimated depths with horizontal adjustments seemed to be more erroneous the further the camera was off centre. With vertical translational movements

Table 8 Elevational and azimuthal effects on estimated cup depth

\begin{tabular}{lll}
\hline $\begin{array}{l}\text { Type of angular } \\
\text { camera adjustment }\end{array}$ & $\begin{array}{l}\text { Actual cup } \\
\text { depth }(\mathrm{mm})\end{array}$ & $\begin{array}{l}\text { Measured cup } \\
\text { depth }(\mathrm{mm}) \\
n=1\end{array}$ \\
\hline Camera in central position & 1.27 & 1.37 \\
Camera rotated right by 1 step & 1.27 & 1.26 \\
Camera rotated right by 2 steps & 1.27 & 1.16 \\
Camera rotated left by 1 step & 1.27 & 1.16 \\
Camera rotated left by 2 steps & 1.27 & 1.37 \\
Camera returned to central position & 1.27 & 1.37 \\
Camera rotated upward 1 step & 1.27 & 0.95 \\
Camera rotated upward 2 steps & 1.27 & 1.37 \\
Camera rotated downward 1 step & 1.27 & 1.58 \\
Camera rotated downward 2 steps & 1.27 & 1.68 \\
\hline
\end{tabular}

Depth sensitivity $=0 \cdot 105 \mathrm{~mm}$.

Table 9 Effect of horizontal and vertical translational movements on estimated cup depth

\begin{tabular}{lll}
\hline $\begin{array}{l}\text { Type of transitional } \\
\text { camera adjustment }\end{array}$ & $\begin{array}{l}\text { Actual cup } \\
\text { depth }(\mathbf{m m})\end{array}$ & $\begin{array}{l}\text { Measured cup } \\
\text { depth }(\mathrm{mm}) \\
n=1\end{array}$ \\
\hline Camera in centered position & 1.27 & 1.26 \\
Camera shifted right $1 \mathrm{~mm}$ & 1.27 & 1.26 \\
Camera shifted right $2 \mathrm{~mm}$ & 1.27 & 0.95 \\
Camera shifted left $1 \mathrm{~mm}$ & 1.27 & 1.37 \\
Camera shifted left $2 \mathrm{~mm}$ & 1.27 & 1.68 \\
Camera returned to central position & 1.27 & 1.37 \\
Camera shifted upward I mm & 1.27 & 1.47 \\
Camera shifted upward $2 \mathrm{~mm}$ & 1.27 & 0.95 \\
Camera shifted downward $1 \mathrm{~mm}$ & 1.27 & 1.37 \\
Camera shifted downward $2 \mathrm{~mm}$ & 1.27 & 1.26 \\
\hline
\end{tabular}

Depth sensitivity $=0.105 \mathrm{~mm}$. 
the errors in measured cup depth did not appear to be associated with the amount of camera movement.

\section{Discussion}

The digital photogrammetric measurements of cup depth in the model eye accurately approximated the actual cup depth except with very deep cups. The larger error on the deeper cups is probably due to poor focus on the vessels at the cup bottom, since the photographs were taken using the retinal surface as the plane of best focus for the photographer. This dilemma might also be encountered in the human eye, in which the cup is so deep that out-of-focus photographs result. It is extremely difficult to overcome this problem. Hence certain limitations on the usefulness of digital photogrammetry in patients with advanced glaucoma and extremely deep cups are created.

The chief source of error in the cup depth estimates resulted from the finite depth sensitivity which in turn derives from the finite pixel spacing of the film digitiser. The depth sensitivities of 0.105 and 0.120 $\mathrm{mm}$ are much poorer than those we are accustomed to when analysing the disc photographs of patients. This reduction in depth sensitivity results from the quadratic dependence of the depth sensitivity on the model eye's focal length, which is approximately 3 times that of the human eye. Since the model eye geometry is 3 times that of the human eye, the poorer depth sensitivity proved acceptable in the cup depth analysis, and changes of $0.53-0.60 \mathrm{~mm}$ could be easily detected with the above sensitivities. What changes will be clinically detectable and significant in patients with chronically elevated intraocular pressure are at present unknown. If one interpolates these data to the human eye, changes of approximately 59-67 $\mu \mathrm{m}$ can easily be measured. What this change represents in terms of cup contour is still a matter of speculation.

Other differences exist in the optics of the model and human eyes. Most notably, the pupil is in front of the cornea in the model eye, and ammetropia is simulated solely by moving the retina rather than by a combination of axial length and corneal curvature. Moreover, we appreciate the fact that the optic cup of the Zeiss model eye is not a perfect facsimile of the human optic nerve head and cup. The absolute symmetrical cylindrical contour with the flat floor to the cup without the subtle topographical contours of the human cup make it a less than perfect model. However, it did provide a means of studying the photogrammetric accuracy with known cup depths.

The errors induced in cup depth measurements with reduction in pupil diameter below $5 \mathrm{~mm}$ indicate that it is important to measure and record the actual diameter of the patient's pupil during stereophotography. Pupils $4 \mathrm{~mm}$ and smaller will not permit either adequate stereophotography with the Donaldson camera or accurate digital photogrammetric evaluation. We must be aware that visit-to-visit variations in pupil size can produce false impressions of progressive change in cup size. If the diameter of the pupil is less than $5.0 \mathrm{~mm}$, correction factors might have to be used to adjust for alterations in cup parameters induced by the pupil size.

The underestimation and overestimation of cup depth with myopia and hyperopia respectively is a more serious problem in terms of accuracy of absolute measurements. We must therefore take them into account when measuring cup depth. High hyperopia will lead to excessively large estimates of cup depth, while high myopia will lead to excessively small estimates. However, such incorrect values will not produce false impressions concerning progressive changes in cup depth or contour. Hence the ability of digital photogrammetry to detect progressive or relative changes in cup topography with chronic elevations in intraocular pressure is not altered by the patient's refractive error. Finally, we must also bear in mind that error in the cup depth measurement tends to increase with increasing myopia.

The addition of a cylinder to the model eye introduces an error into the measurement of cup depth. This error is noted only when the cylindrical axis is at $90^{\circ}$. With a cylinder axis at $180^{\circ}$ the error is within the limits of the analysis. Moreover, the introduced error is related to the power of the cylinder, i.e., a plus cylinder causes an underestimation of cup depth and a minus cylinder causes overestimation. An explanation for these observations is as follows. The cylinders together with the camera lens in effect form a simple Galilean telescope, but they produce linear magnification or reduction in only one meridian, namely, the meridian perpendicular to the axis. This distortion produces an apparent (but not real) change in the cup depth. In this system a plus cylinder axis $90^{\circ}$ in the Galilean system will magnify linearly along $180^{\circ}$. Since the depth is measured by parallax using points along the horizontal axis, it will appear smaller relative to this elongation of the horizontal axis by the plus cylinder axis $90^{\circ}$. The converse would be true with the minus cylinder axis $90^{\circ}$. One would not expect cylinders axis $180^{\circ}$ to alter the computer's depth analysis, since they will not magnify the points along the horizontal axis.

We feel confident that simple photographic manoeuvres, for example, refocusing, realignment, and repositioning, should not materially affect the 
accuracy of the digital photogrammetric technique. However, more complex manoeuvres such as elevational and azimuthal rotations of the camera as well as horizontal and vertical translational movement can alter the accuracy of the technique. The complex manoeuvres of elevation and translation are very difficult to perform with the Donaldson camera and particularly in this situation, where a model eye is securely mounted to the headrest. However, in a clinical setting (and with other types of fundus cameras) the equivalent of these complex manoeuvres may be performed by the patient through either faulty positioning in the headrest or faulty fixation. Such malalignments of the camera system with the eye's axis, are therefore possible in the clinical setting, and great precaution should be taken to avoid them.

Since the photogrammetric procedure in the model eye examines only a small region of the optic disc and border, averages measurements over only 2 depth planes, and uses an artificial fundus pattern, the accuracy of cup depth measurement from photogrammetric analyses of optic cups in the human eye cannot be intuitively expected. Methods of determining actual cup depth in the human optic nerve head must be developed so that comparison with photogrammetric estimates can be undertaken before one can feel confident that digital photogrammetric estimates of cup depth from stereophotographs of human optic cups are accurate.

We thank Dr Michael Pinnolis, Mrs M. Crane, and Mr J. L. Hopkins for their help in preparing this manuscript.

This work was supported by research grant EY 01248 of the National Eye Institute, National Institutes of Health (Dr Rosenthal).

\section{References}

1 Kottler MS, Rosenthal AR, Falconer DG. Digital photogrammetry of the optic nerve head. Invest Ophthalmol Visual Sci 1974; 13: 116-20.

2 Rosenthal AR, Kottler MS, Donaldson DD, Falconer DG. Comparative reproducibility of the digital photogrammetric procedure utilizing three methods of stereophotography. Invest Ophthalmol Visual Sci 1977; 16: 54-60.

3 Donaldson DD. A new camera for stereoscopic fundus photography. Trans Am Ophthalmol Soc 1964; 62: 429-58.

4 Falconer DG, Rosenthal AR. Digital photogrammetry on the Zeiss model eye. Computers in Ophthalmology. IEEE Computer Society Proceedings April 1979: 145-52. 